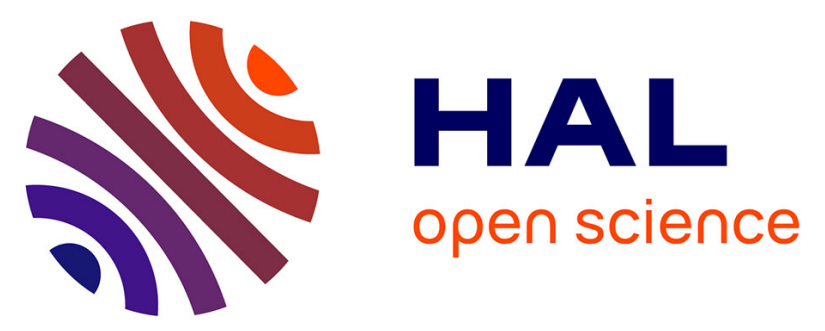

\title{
Experimental investigation about energy balance of electron emission from materials under electron impacts at low energy: application to silver, graphite and $\mathrm{SiO} 2$
}

M. Villemant, P. Sarrailh, M. Belhaj, Laurent Garrigues, C. Boniface

\section{- To cite this version:}

M. Villemant, P. Sarrailh, M. Belhaj, Laurent Garrigues, C. Boniface. Experimental investigation about energy balance of electron emission from materials under electron impacts at low energy: application to silver, graphite and SiO2. Journal of Physics D: Applied Physics, 2014, 50 (48), 10.1088/1361-6463/aa91af . hal-01716166

\author{
HAL Id: hal-01716166 \\ https://hal.science/hal-01716166
}

Submitted on 23 Feb 2018

HAL is a multi-disciplinary open access archive for the deposit and dissemination of scientific research documents, whether they are published or not. The documents may come from teaching and research institutions in France or abroad, or from public or private research centers.
L'archive ouverte pluridisciplinaire HAL, est destinée au dépôt et à la diffusion de documents scientifiques de niveau recherche, publiés ou non, émanant des établissements d'enseignement et de recherche français ou étrangers, des laboratoires publics ou privés. 


\title{
Experimental investigation about Energy balance of electron emission from materials under electron impacts at low energy: application to silver, graphite and $\mathrm{SiO}_{2}$
}

\author{
M Villemant ${ }^{1}$, P Sarrailh ${ }^{1}$, M Belhaj ${ }^{1}$, L Garrigues $^{2}$, C \\ Boniface $^{3}$ \\ ${ }^{1}$ ONERA - The French Aerospace Lab, 31055 CEDEX, FRANCE \\ ${ }^{2}$ LAPLACE Laboratory, Toulouse University, CNRS, INPT, UPS, FRANCE \\ ${ }^{3}$ CNES - French National Space Agency, 18 Avenue Edouard Belin, F-31401 \\ Toulouse, FRANCE \\ E-mail: marc.villemant@onera.fr \\ August 2017
}

\begin{abstract}
.
The energy balance measurement of electron emission at a wall submitted to electron impact at low incident energy is a topic of interest for miscellaneous technological applications. This article points out the experimental protocol, biases corrections and post-process needed to obtain reproducible and quantitative electron emission measurements. The measurements have been performed for incident electrons energy between $5 \mathrm{eV}$ and $105 \mathrm{eV}$ and for three samples materials: silver, graphite and $\mathrm{SiO}_{2}$. These measurements show that wall absorbs more energy at high incident electrons energy and that graphite absorbs more energy than silver, than $\mathrm{SiO}_{2}$. Results are presented for mono-energetic incident electron beam and for a Lambertian energy distribution. Analytical laws fitted from experimental results and applicable for modelling issue are proposed for a Lambertian distribution of incident electrons.
\end{abstract}

Keywords: Electron Emission, Energy balance, Hall Thruster, plasma modelling

Submitted to: J. Phys. D: Appl. Phys. 


\section{Nomenclature}

\subsection{Acronyms}

- BSE: Backscattered electrons

- EBSE: Elastically Backscattered Electrons

- EE: Electron Emission

- EEA: Electron Energy hemispherical Analyser

- EEED: Emitted Electron Energy Distribution

- IBSE: Inelastically Backscattered Electrons

- HT: Hall Thruster

- PIC: Particle-In-Cell

- SE: Secondary Electrons

- TEEY : Total Electron Emission Yield

\subsection{Roman characters}

- $E_{a}$ : Energy of electrons arriving at the analyser $[\mathrm{eV}]$

- $E_{e}$ : Emitted electron energy [eV]

- $\left[E_{e}\right]$ : Mean emitted electron energy [eV]

- $E_{g}$ : Incident electron energy at the electron gun $[\mathrm{eV}]$

- $E_{0}$ : Incident electron energy $[\mathrm{eV}]$

- $\mathcal{E}_{a b s}$ : Total energy absorbed by the wall [eV]

- $\mathcal{E}_{e}$ : Total kinetic energy of electrons emitted by the wall $[\mathrm{eV}]$

- $\mathcal{E}_{0}$ : Total kinetic energy of incident electrons to the wall $[\mathrm{eV}]$

- $d$ : The diameter of the acceptance equivalent surface $[\mathrm{mm}]$

- $h$ : The distance between the sample and the electron energy analyser [mm]

- $R_{E}$ : Energy efficiency of electron/material interaction $[\varnothing]$

\subsection{Greek characters}

- $\Gamma$ : Electron flux $\left[\mathrm{m}^{-2} \mathrm{~s}^{-1}\right]$

- $\Gamma_{0}$ : Incident electron flux $\left[\mathrm{m}^{-2} \mathrm{~s}^{-1}\right]$

- $\Gamma_{e}$ : Emitted electron flux $\left[\mathrm{m}^{-2} \mathrm{~s}^{-1}\right]$

- $\Gamma_{a}$ : Electron flux collected by the analyser $\left[\mathrm{m}^{-2} \mathrm{~s}^{-1}\right]$

- $\delta \Gamma$ : Normalised electron energy distribution function $\left[\mathrm{eV}^{-1}\right]$

- $\delta \Gamma_{e}$ : Normalised electron energy distribution function $\left[\mathrm{eV}^{-1}\right]$

- $\delta \Gamma_{0}$ : Normalised electron energy distribution function $\left[\mathrm{eV}^{-1}\right]$

- $\delta \Gamma_{\mathcal{L}, T_{e}}$ : Normalised energy Lambertian distribution function $\left[\mathrm{eV}^{-1}\right]$
- $\sigma$ : Total electron emission yield $[\varnothing]$

- $\varphi$ : Surface potential bias [V]

\section{Introduction}

Under electron impacts, electrons are emitted from the surface and near surface region of materials. This phenomenon is called electron emission induced by electrons (EE). The emitted electrons are secondary electrons (SE) and backscattered electrons (BSE).

Until nowadays, EE has been thoroughly studied for high incident electrons energy $\left(E_{0}>100 \mathrm{eV}\right)[1-8]$. However it has infrequently been studied for $E_{0}<$ $100 \mathrm{eV}$, although this phenomenon has a determining impact on numerous applications such as RF hardware's to prevent multipactor effect [9], electron-cloud effect in particles accelerator [10 or on Hall thrusters (HT) plasma/wall interactions.

Indeed EE is suspected to have a non-negligible impact on HT performance since it has been experimented that changing wall materials affects the thruster operation 11. Moreover it has been evaluated that the majority of energy losses in HT plasma is due to the interaction between the electrons and the wall [12]. Besides, fluid/hybrid models have to consider electron energy losses due to EE at the walls to obtain reasonable electron energy in the thruster channel 13, 14. Moreover, despite the fact that $R_{E}$ is a key parameter in many applications and in particular for HT modelling it is not well characterized. Besides, according the used HT models there is no clear and consensual picture of its value and evolution as a function of the incident electron energy.

In previous articles, the electron energy distribution was used to characterize EE and in particular to evaluate the $\mathrm{BSE} / \mathrm{SE}$ ratio $8,15,16$. In addition, the link between EE and electron transport in the context of HT has been studied through Particle-In-Cell (PIC) models 17,20 and all the studies have pointed out that not only the number of emitted electrons is important but also energy carried by emitted electrons. Furthermore, several particles models have been created to improve the modelling of the EE influence on HT plasma behaviour. Nonetheless their conclusions diverge in part because of the accuracy of the EE models. Thus, it is important to have accurate electrons energy distribution measurement to assess EE models accuracy in these simulations. Besides, emitted electrons energy distribution measurement is also critical to calculate accurately the multipactor triggering in satellites RF hardware's.

So far, numerous papers have investigated the electron emission yields experimentally $[21,22$ and theoretically 23 but the literature suffers from a lack of information about electrons energy losses at the walls. 
In this article, EE interaction has been studied from an energy balance point of view. The objective is to measure the energy balance of an electron flux at a wall for several materials and several incident electron energies $E_{0}$. The energy efficiency of electron/wall interaction $\left(R_{E}\right)$ is defined as the kinetic energy carried by electrons re-emitted in the plasma from the wall divided by the kinetic energy carried by incident electrons impacting on the wall:

$$
R_{E}=\frac{\mathcal{E}_{e}}{\mathcal{E}_{0}}=\frac{\mathcal{E}_{0}-\mathcal{E}_{a b s}}{\mathcal{E}_{0}}=1-\frac{\mathcal{E}_{a b s}}{\mathcal{E}_{0}}
$$

With $\mathcal{E}_{0}[\mathrm{eV}]$ the kinetic energy of incident electrons to the wall, $\mathcal{E}_{e}[\mathrm{eV}]$ the kinetic energy of electrons emitted by the wall, and $\mathcal{E}_{a b s}[\mathrm{eV}]$ the total energy absorbed by the wall. The higher $R_{E}$, the higher the power reflected by the wall is. $R_{E}=1$ corresponds to an incident kinetic energy fully reflected to the vacuum and $R_{E}=0$ corresponds to an incident kinetic energy fully absorbed by the wall.

$R_{E}$ assessment implies to measure both the total electron emission yield (TEEY) and the emitted electron energy distribution (EEED).

This article emphasizes on methodology used to correct EEED measurement biases at low energy. Indeed, the extraction of the EE properties from one of the measured spectra is far from being obvious as the electron transport between the sample and the analyser and electrons collection measurement depend on electrons energy and sample voltage. It is shown in this work that several corrections are needed to get reliable EEED at low $E_{0}$. For this purpose, a dedicated experimental protocol and data processing methodology were developed in order to extract $R_{E}$.

This article is organized in three parts. First of all the experimental protocol is presented in section 3 . In section 4, the need for EEED measurement correction and post-processing is highlighted and corrections and post-processes methods are described. It is shown that electron transport between sample and analyser, analyser energy transfer function and sample charging should be assessed to get quantitative measurements. In section 5 measurements results are presented. The energy efficiency $R_{E}$ as a function of the incident electron energy is determined for $\mathrm{SiO}_{2}$, graphite and silver samples. It is shown that the energy absorption increases with the impact energy of the incident electrons and that the $R_{E}$ is highly dependent on the wall material composition. A fit of $R_{E}$ evolution as a function of the incident electron energy is given for a Lambertian energy distribution of incident electrons to be used in modelling. Finally in section 6, the results are summarised.

\section{Experimental protocol}

Two kinds of experimental data are needed to calculate $R_{E}$ : the Total Electron Emission Yield (TEEY) and the Emitted Electron Energy Distribution (EEED). They have been measured for $E_{0}$ between $5 \mathrm{eV}$ and $105 \mathrm{eV}$ with an energy step of $5 \mathrm{eV}$.

\subsection{Theoretical elements definition}

TEEY is defined as the number of emitted electrons divided by the number of incident electrons:

$$
\sigma\left(E_{0}\right)=\frac{\Gamma_{e}\left(E_{0}\right)}{\Gamma_{0}\left(E_{0}\right)}
$$

Where $\Gamma_{e}\left[\mathrm{~m}^{-2} \mathrm{~s}^{-1}\right]$ is the emitted electrons flux from the wall, $\Gamma_{0}\left[\mathrm{~m}^{-2} \mathrm{~s}^{-1}\right]$ is the incident electrons flux to the wall and $E_{0}[\mathrm{eV}]$ the incident electron energy. Both $\Gamma_{0}$ and $\Gamma_{e}$ depend on $E_{0}$.

In order to evaluate the energy balance at the wall, both the incident and emitted electrons flux are needed, but also their energy distribution. To describe them, the normalised energy distribution functions of electron fluxes are defined as:

$$
\delta \Gamma(E)=\frac{1}{\Gamma} \frac{d \Gamma}{d E} \quad\left[\mathrm{eV}^{-1}\right]
$$

These functions depend on the incident electrons energy. The normalized energy distribution function of electron flux is noted $\delta \Gamma_{0}(E)$ for incoming electrons to the wall, and $\delta \Gamma_{e}(E)$ for emitted electrons from the wall.

These normalized quantities can be written as:

$$
\int_{0}^{+\infty} \delta \Gamma(E) \cdot d E=1
$$

\subsection{Measurement principle}

From definition written in eq, 1 , the energy efficiency is obtained from the ratio of the mean energy of emitted and collected electrons that can be written as:

$$
R_{E}\left(E_{0}\right)=\frac{\Gamma_{e} \int_{0}^{+\infty} E \cdot \delta \Gamma_{e}\left(E, E_{0}\right) \cdot d E}{\Gamma_{0} \int_{0}^{+\infty} E \cdot \delta \Gamma_{0}\left(E, E_{0}\right) \cdot d E}
$$

As the incident electron beam is quasi-monocinetic (energy spread $0.8 \mathrm{eV}[24]$ ), the expression of $R_{E}$ can be simplified to:

$$
R_{E}\left(E_{0}\right)=\sigma\left(E_{0}\right) \frac{\left[E_{e}\right]}{E_{0}}
$$

Where $\sigma\left(E_{0}\right)$ is the TEEY of the sample and $\left[E_{e}\right]$ is the mean energy of emitted electrons $[\mathrm{eV}]$ computed from EEED measurement as:

$$
\left[E_{e}\right]=\int_{0}^{+\infty} E \cdot \delta \Gamma_{e}(E) . d E
$$


EEED measurements give an approximation of energy distribution:

$$
\delta \Gamma_{e}\left(E, E_{0}\right) \simeq \frac{1}{\sum_{i=1}^{N} N\left(E_{i}\right)} \frac{N\left(E_{i}\right)}{\Delta E_{i}}
$$

With $N\left(E_{i}\right)$ [cts/s] the number of electrons counted by the analyser between the energies $E_{i}$ and $E_{i}+\Delta E_{i}$. The smaller the energy step, the better this approximation is. data as:

$\left[E_{e}\right]$ can then be approximated from experimental

$$
\left[E_{e}\right] \simeq \frac{\sum_{i=1}^{N} N\left(E_{i}\right) \cdot E_{i} \cdot \Delta E_{i}}{\sum_{i=1}^{N} N\left(E_{i}\right) \cdot \Delta E_{i}}
$$

\subsection{Experimental set-up description}

Experiments have been performed at low incident energy $\left(E_{0}<100 \mathrm{eV}\right)$, at low incident electrons current density (few $\mu \mathrm{A} / \mathrm{cm}^{2}$ ) and in Ultra-High Vacuum Chamber, at vacuum level of $10^{-8}$ mbar. The used electron gun is a Kimball instruments ELG-2 with very low energies capabilities $(1 \mathrm{eV}-2 \mathrm{keV})$. The electron energy hemispherical analyser (EEA) is an Omicron Nanotechnology EA125. The samples were placed face to electron gun and at $45^{\circ}$ angle with respect of the analyser axis (cf. FIG 1). The input analyser diaphragm is placed at $30 \mathrm{~mm}$ from the sample surface. TEEY and emitted electrons energy spectra are measured for $E_{0}$ between $5 \mathrm{eV}$ and $105 \mathrm{eV}$. The detail of the measurement protocol of the TEEY was described in 21. An energy step of $0.2 \mathrm{eV}$ has been used to record the EEED. The TEEY and the emitted electrons spectra were measured for three different materials: silver, graphite and $\mathrm{SiO}_{2}$. The $\mathrm{SiO}_{2}$ sample has been produced by plasma deposition on metal substrate. Thus it is an amorphous sample with a width below $100 \mathrm{~nm}$.

Furthermore, several calibrations must be proceeded in order to have suitable EE measurements.

The incident electron beam presents a small divergence. Thus, it is checked that the whole incident electrons flux encounters the sample and not the sample holder in order not to measure a mix between sample and sample holder EE. Size of the incident electrons spot has been evaluated with a Faraday cup to ensure that incident electrons could only hit the sample and not the sample holder.

A significant part of emitted electrons has a very low energy (lower than $1 \mathrm{eV}$ ) when they are emitted from the sample surface. Due to electrostatic configuration of the experimental set-up, they could go back to the sample. Consequently both TEEY and EEED could be under-evaluated at low energy. Vacuum chamber internal shell has been electrically grounded and the sample holder has been negatively biased with
Electron Gun

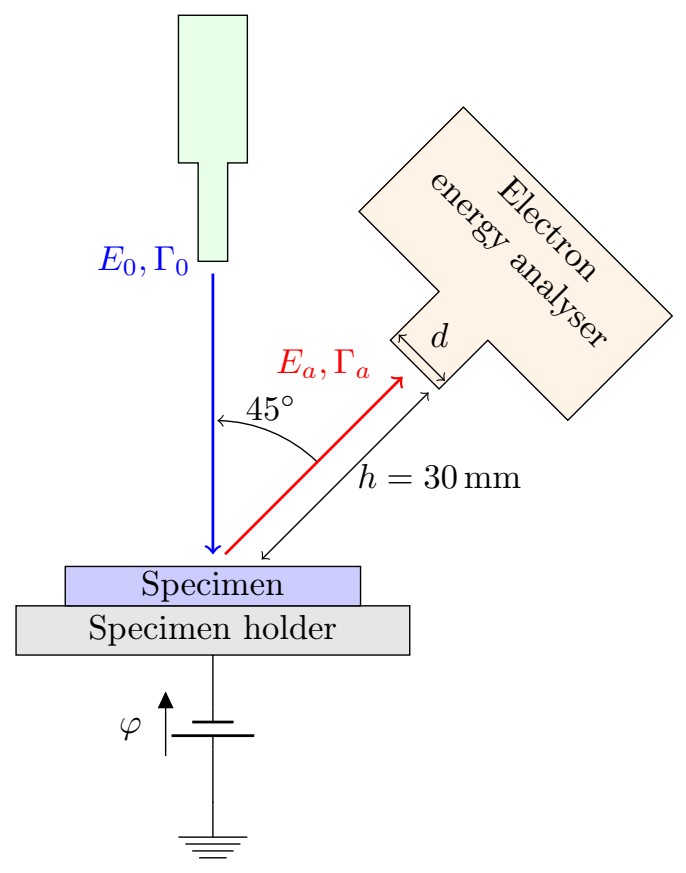

Figure 1: Scheme of experimental set-up

a static negative potential between $-5 \mathrm{eV}$ and $-15 \mathrm{eV}$ in order to prevent sample from recollecting low energy emitted electrons and the tertiaries electrons emitted from the shells. This offset has an impact on the measured EEED which has to be corrected (cf. section 4.2.1 and EEED peaks definition).

\section{Measurements corrections and post-process}

\subsection{Highlighting of measurement uncertainties}

By observing the raw measured spectra, it is possible to state that several corrections are needed to get accurate and reproducible results. First of all, it can be observed that raw energy spectra present an offset due to the surface potential whereas it is known that experimental secondary electrons energy distribution onset is situated close to $1 \mathrm{eV}$ referring to vacuum level [25]. To assess the surface potential effects on the EEED shape, electrons energy spectra and $R_{E}\left(E_{0}\right)$ have been measured for three different biases $(\varphi=-5 \mathrm{eV}, \varphi=-10 \mathrm{eV}$ and $\varphi=-15 \mathrm{eV}$ ) with keeping the incident electron energy fixed. Indeed, the incident electron energy at the electron gun $\left(E_{g}[\mathrm{eV}]\right)$ has been increased so that:

$$
E_{g}=E_{0}+e|\varphi|
$$

It can be stated that the real energy distribution of emitted electrons should not depend on surface potential after correction of the surface potential offset. 


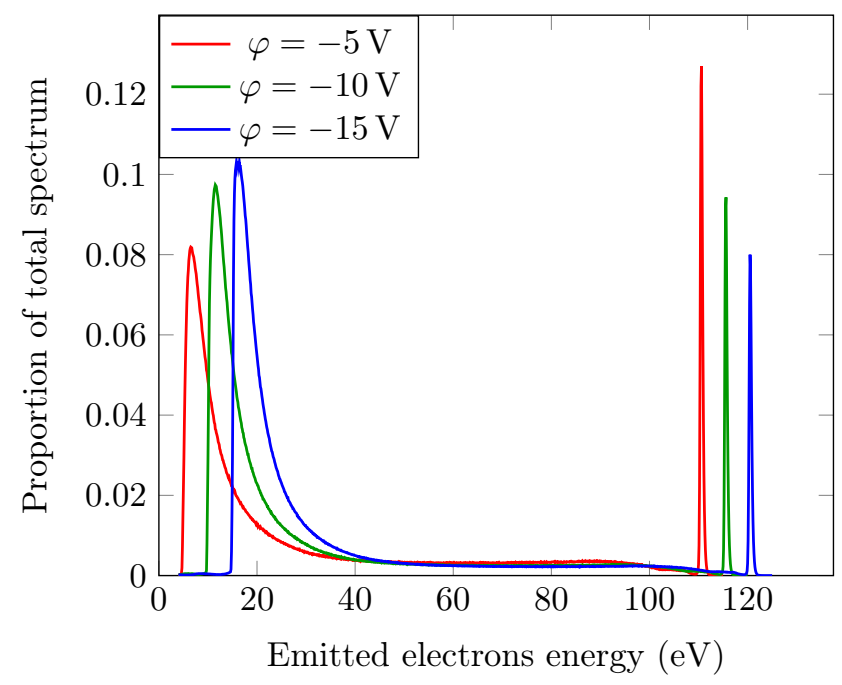

Figure 2: Measured electron energy distribution on silver specimen with $E_{0}=105 \mathrm{eV}$, for three biases before correction

Nonetheless, it can be observed on the experimental spectra shown in FIG.2 that, in addition to the offset due to potential biases, the three spectra also differ in term of proportions. It can be seen in FIG 2 that for $\varphi=-5 \mathrm{eV}$, the low energy peak is lower than the high energy peak, for $\varphi=-10 \mathrm{eV}$, the low energy peak is as high as the high energy peak and that and for $\varphi=-15 \mathrm{eV}$, the low energy peak is higher than the high energy peak (cf. 5.1 for EEED description).

These discrepancies on the spectrum are due to the electrostatic configuration in the chamber. The electric field created in the vacuum by the sample biasing affects the electron analyzer collection. This effect depends on the emitted electrons energy at the sample surface (cf. section 4.2). Besides, the number of electrons counted by analyser is highly dependent on the electron energy at the analyser entrance as it will be shown in section 4.2.2 To address this shortcoming a specific correction methodology is used to measure EEED and extract $R_{E}$ from measurements. The correction proposed and their impacts on measurements are described in the next section.

\subsection{Measurements post-processing}

4.2.1. Electrons transports between sample and analyser, surface potential influence During the electron energy distribution measurement, the sample holder is negatively biased in order to prevent the recollection of low energy secondary electrons [16]. This surface potential creates an offset on measured EEED which should be subtracted to get the real EEED. An other undesirable consequence of this biasing is

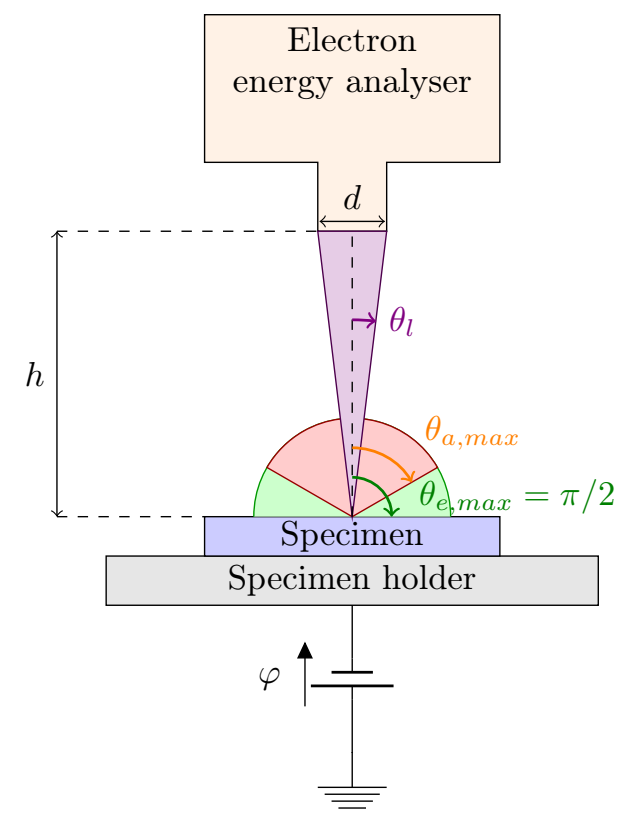

Figure 3: Geometrical scheme of electrons transport between specimen and analyser

that the created electric field into the vacuum may distort the emitted electrons angular distribution [26] and consequently, the electron energy distribution measurement (electron collection efficiency of the analyser).

If an isotropic electron emission is assumed which is reasonable at low energy - it can be observed that the electron flux reaching the analyser entrance increases when the surface potential is increased. As the surface potential will become more negative, emitted electrons will be repulsed by the sample and focused on the analyser (cf. FIG 3). Consequently, the more negative the surface potential, the higher collected flux by the analyser is.

The transfer function of the transport between the sample surface and the analyser will be dependent on surface potential and geometrical aspect (distance between sample and the analyser, diameter of the acceptance equivalent surface, etc.). This angular distribution distortion is corrected by taking into account the approximation of isotropic angular distribution of EE. This transfer function calculates the increase of measured electrons intensity depending on maximum emission angle before and after applying the sample holder bias $\left(\theta_{e, \max }\right.$ and $\left.\theta_{a, \max }\right)$ and the field of view of the analyzer $\left(\theta_{l}\right.$, cf. FIG 3$)$. The transfer 
function is given by:

$H_{T}\left(E_{e}, \varphi\right)=\frac{\Gamma_{a}\left(E_{e}, \varphi\right)}{\Gamma_{e}} \begin{cases}\frac{\arctan \left(\frac{d}{2 h}\right)}{\arctan \left(\sqrt{\frac{E_{e}}{e|\varphi|}}\right)} & \text { for } E_{e}>\frac{d^{2}}{4 h^{2}} e|\varphi| \\ 1 & \text { for } E_{e}<\frac{d^{2}}{4 h^{2}} e|\varphi|\end{cases}$

With:

- $\Gamma_{e}$ : The electron flux emitted by the sample $\left[\mathrm{m}^{-2} \mathrm{~s}^{-1}\right]$

- $\Gamma_{a}$ : The electron flux collected by the analyser $\left[\mathrm{m}^{-2} \mathrm{~s}^{-1}\right]$

- $h$ : The distance between the sample and the electron energy analyser [mm]

- $d$ : The diameter of the acceptance equivalent surface $[\mathrm{mm}]$

- $E_{e}$ : The emitted electron energy $[\mathrm{eV}]$

- $\varphi$ : The bias $[\mathrm{V}]$

- $e$ : The elementary charge $\left[1.6 \times 10^{-19} \mathrm{C}\right]$

Electrons transport transfer function is plotted in FIG 4 for $\varphi=-10 \mathrm{~V}, d=30 \mathrm{~mm}$ and $h=30 \mathrm{~mm}$. This transfer function influences mainly the energy distribution of low energy emitted electrons. It can be observed on this figure that below a minimum energy $E_{e, \min }=\frac{d^{2}}{4 h^{2}} e \varphi, H_{T}\left(E_{e}, \varphi\right)=1$. This means that for $E_{e}<E_{e, \min }$, due to the electric field resulting from the surface charging and directed towards the analyser, all the emitted electrons are caught by the analyser. As $E_{e}$ rises, the resulting electric field is less and less able to redirect all the emitted electrons to the analyser. Thus a decrease of $H_{T}\left(E_{e}, \varphi\right)$ can be observed for intermediate values of $E_{e}$. Finally, for high $E_{e}$, the resulting electric field is too weak to notably influence emitted electrons trajectories and thus $H_{T}\left(E_{e}, \varphi\right)$ tends to a constant value: $H_{T}\left(E_{e}, \varphi\right) \underset{E_{e} \rightarrow+\infty}{\longrightarrow} \frac{2}{\pi} \arctan \left(\frac{d}{2 h}\right)$.

4.2.2. Analyser calibration Quantitative data are needed on emitted electrons spectra and the given EEA built in calibration is not meant to be used at very low incident energy $(<300 \mathrm{eV})$. It has been observed that for the same emitted electrons flux the measured EEA signal $[$ counts $/ \mathrm{s}$ ] will increase with emitted electrons energy. In order to get quantitative measurement, this deviation has been experimentally corrected. For this purpose, the electron gun has been placed facing the analyser and the EEA signal has been measured for different electrons energies between 5 and $300 \mathrm{eV}$ and at known values of electron flux (cf. FIG 5).

FIG 6 shows the obtained electron energy distribution in this configuration. EEED have been centred on $0 \mathrm{eV}$ by subtracting $E_{0}$ set point. Thus, FIG 6 shows EEED deviation to set point. FIG.7 represents the EEED deviation to set point as a function of $E_{0}$. It

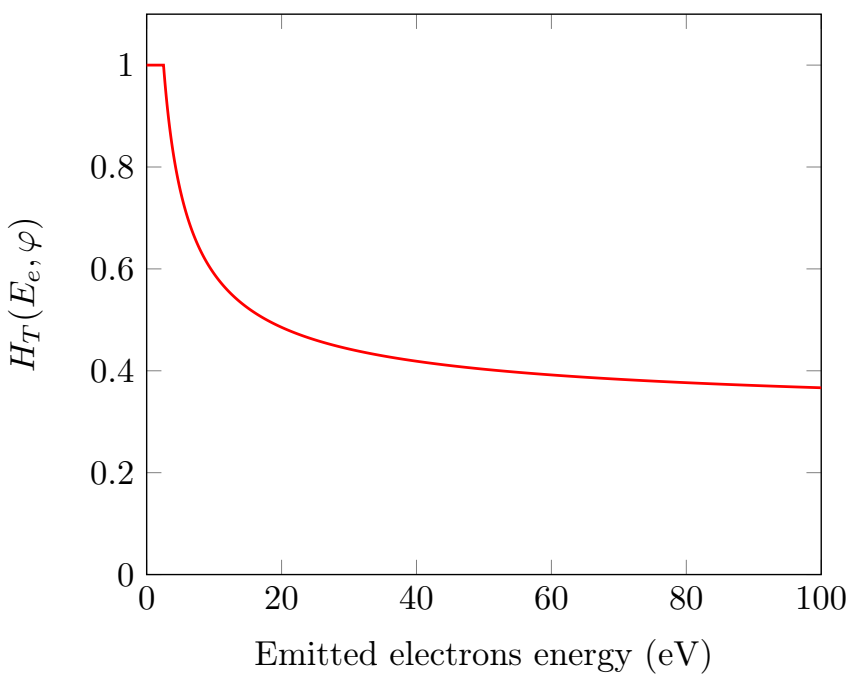

Figure 4: Calculated electron transfer function of transport between the specimen and the electron energy analyzer

\section{Hemispherical analyser}

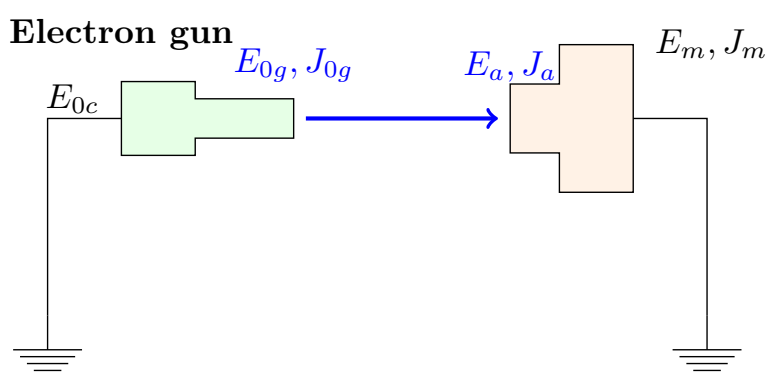

Figure 5: Scheme of analyser calibration set-up

can be noticed on this figure that deviation between $E_{0}$ setting and actual $E_{0}$ is lower than $2 \mathrm{eV}$ for the whole range of $E_{0}\left(E_{0} \in[5 \mathrm{eV} ; 105 \mathrm{eV}]\right)$. Indeed, the maximum deviation to setting is observed for $E_{0}=260 \mathrm{eV}$ with a mean deviation of $1.29 \mathrm{eV}$ and a half value width of $0.58 \mathrm{eV}$.

The analyser transfer function has been defined as:

$$
H_{a}\left(E_{0}\right)=\frac{I_{0}}{I_{\max }}
$$

Where $I_{0}[$ counts/s] is the measured electrons signal at the incident electrons energy and $I_{\max }$ [counts/s] is the measured electrons signal at the maximum experimental incident electrons energy (here, at $E_{0}=130 \mathrm{eV}$, cf.FIG 8). $I_{\max }$ has been chosen for practical reasons: it allows treating only $H_{a}$ values between 0 and 1 . Besides, as only normalized values of EEED are considered (cf. eq4), $I_{\max }$ choice has no influence on EEED and values. For practical reasons, 


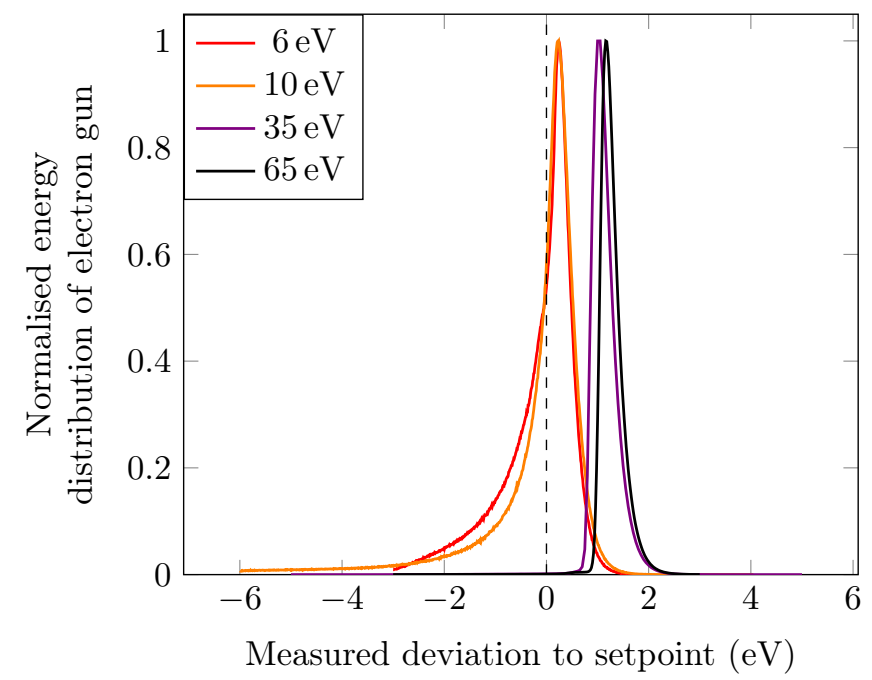

Figure 6: Measured electron gun deviation to $E_{0}$ set point

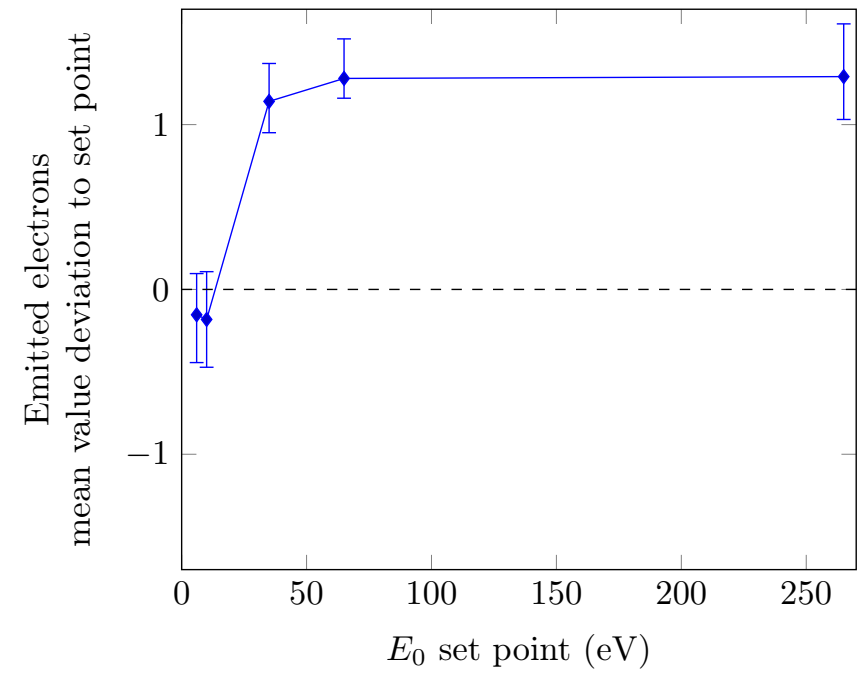

Figure 7: Difference between actual electrons emitted mean energy and set point value

$H_{a}\left(E_{a}\right)$ has been fitted using the following expression:

$$
H_{a}\left(E_{a}\right)=A \cdot e^{\alpha E_{a}}
$$

With: $A=0.0192$ and $\alpha=-0.032 \mathrm{eV}^{-1}$.

Thus, the whole transfer function of the system can be written:

$$
H\left(E_{e}, \varphi\right)=H_{T}\left(E_{e}, \varphi\right) \cdot H_{a}\left(E_{e}+\varphi\right)
$$

In order to correct this variation, measured electron fluxes should be divided by the transfer function before

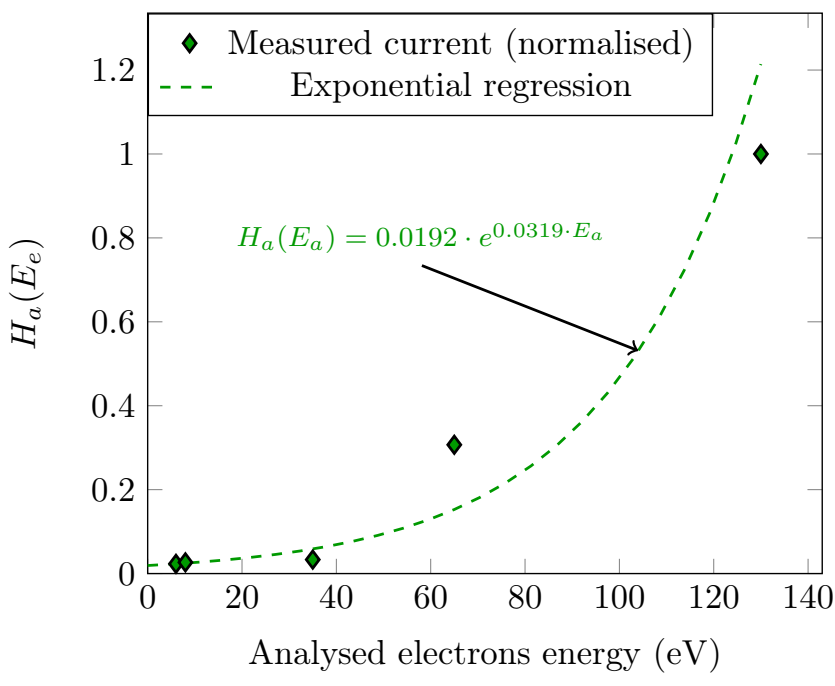

Figure 8: Analyzer EA125 transfer function (normalized), points are experimental data [27], doted line is the exponential regression obtained from equation (13)

being used. Thus eq 5 is corrected according to:

$$
\delta \Gamma_{e}\left(E_{i}\right) \simeq \frac{1}{\sum_{i=1}^{N}\left(\frac{N\left(E_{i}\right)}{H\left(E_{i}, \varphi\right)}\right)} \times \frac{N\left(E_{i}\right)}{H\left(E_{i}, \varphi\right) \Delta E_{i}}
$$

4.2.3. Dielectric sample charging under electron irradiation In order to minimize the charging effects on dielectric material, samples have been chosen as thin as possible (tens of $\mathrm{nm}$ for $\mathrm{SiO}_{2}$ used here). Nevertheless, a small static charge remains. As the onset spectra of emitted electrons start at approximately $0 \mathrm{eV}$, it is possible to correct the energy offset after measurement by subtracting the energy at which the EEED curve take off. By using this method, it is also possible to measure the surface potential variation as a function of incident electron energy (cf. Fig 9). Thus it can be observed that surface potential of $\mathrm{SiO}_{2}$ sample fluctuates between $+4 \mathrm{~V}$ and $-2 \mathrm{~V}$ around the set bias $\varphi$ depending on the incident electron energy $E_{0}$. It can be observed that, even at the highest surface potential, $V$ still remains negative $\left(V=-5.3 \mathrm{~V}\right.$ for $\left.E_{0}=68.7 \mathrm{eV}\right)$. Thus it is clear, that no recollection can occur in these conditions.

\subsection{Correction influence on EEED and $R_{E}$}

This calibration has been checked by measuring emitted electrons spectra with biasing the sample holder of the silver sample by three different negative potentials $(-5 \mathrm{eV},-10 \mathrm{eV}$ and $-15 \mathrm{eV})$ and adjusting the accelerating voltage of the electron gun in such a way that the incident electron arriving energy at the surface was kept unchanged. Variation between 


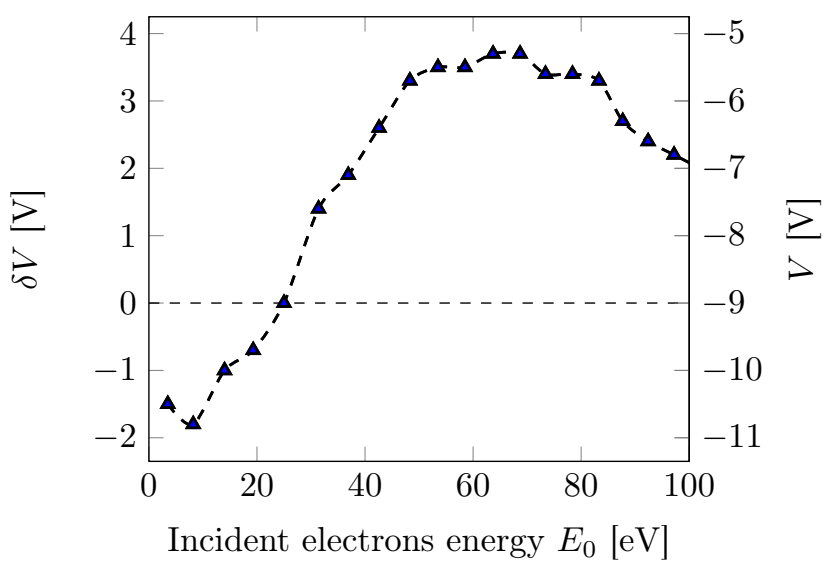

Figure 9: Surface potential $V[\mathrm{~V}]$ and surface potential variation $\delta V[\mathrm{~V}]$ of the $\mathrm{SiO}_{2}$ specimen as a function of $E_{0}$

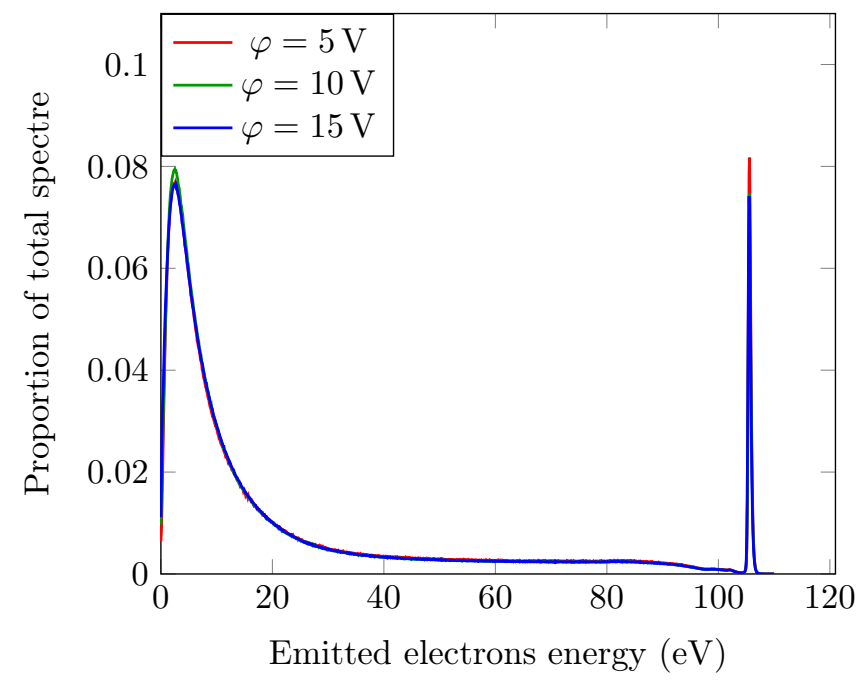

Figure 10: Measured electron energy distribution on silver specimen with $E_{0}=105 \mathrm{eV}$ for three biases, in the same experimental conditions as FIG,2, after transfer function correction. Impact of transfer functions correction on spectra measurement

FIG 2 and FIG 10 shows a significant reduction of the measured EEED discrepancies. Indeed, contrary to FIG 2 (without corrections) where three different offset can be observed for the three measured EEED and where their proportions don't match, it can be observed in FIG 10 that the three measured EEED are superimposed and that all the EEED curves start at $0 \mathrm{eV}$.

Moreover $R_{E}\left(E_{0}\right)$ has been calculated depending on surface voltage and is represented in FIG 11 before (FIG 11a) and after correction (FIG,11b). FIG 11a shows that, without correction and post-processing,
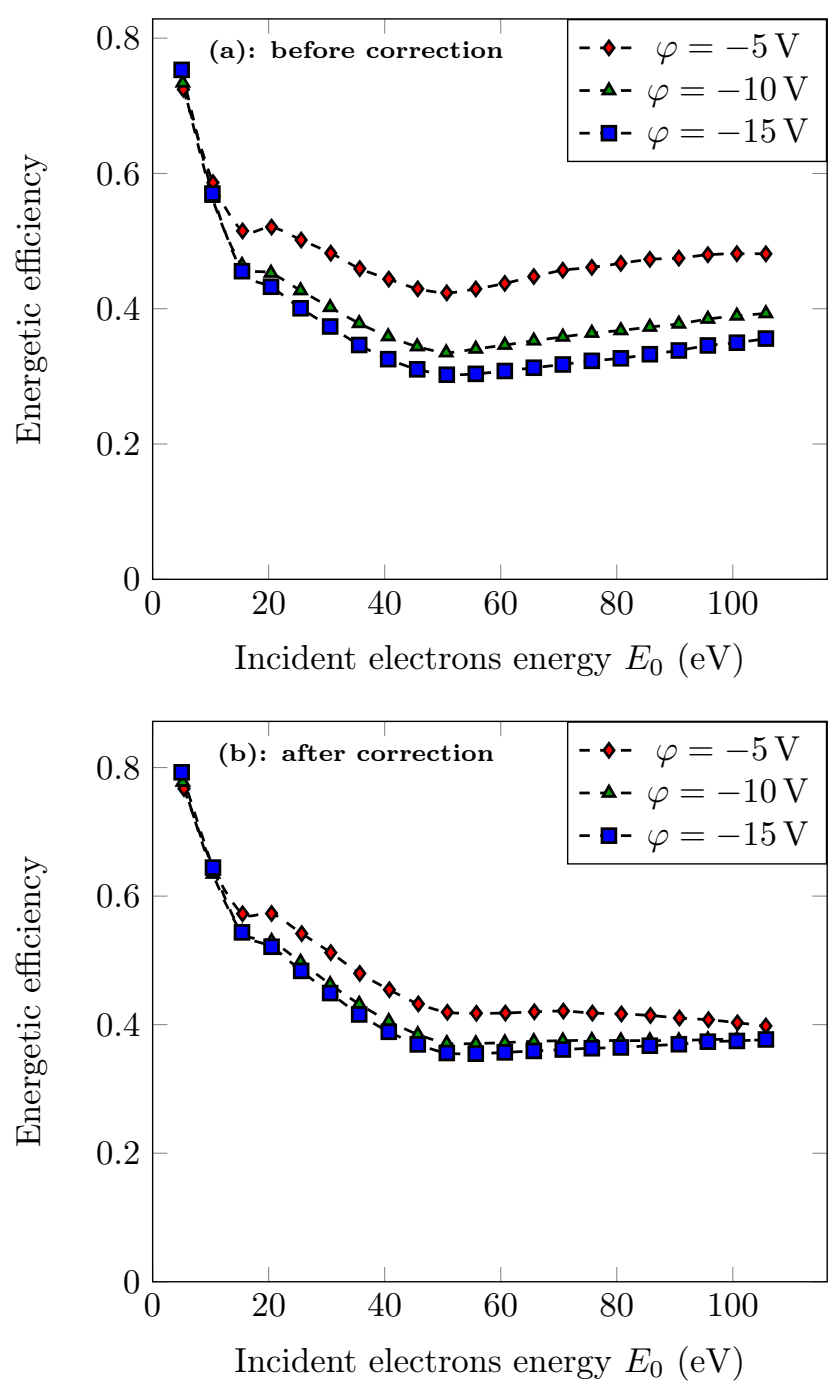

Figure 11: $R_{E}\left(E_{0}\right)$ measurement depending on surface charging for silver specimen. These data have been taken before (a) and after (b) EA transfer function correction

a non-negligible deviation exists between $R_{E}\left(E_{0}\right)$ measurements depending on $\varphi$. This deviation is negligible at low energy but becomes significant at high energy. For example, for $E_{0}=105 \mathrm{eV}$, there is a $12.5 \%$ difference between $R_{E}\left(E_{0}\right)$ measurements for $\varphi=-5 \mathrm{~V}$ and $\varphi=-15 \mathrm{~V}$ and $8.8 \%$ difference between $R_{E}\left(E_{0}\right)$ measurement for $\varphi=-10 \mathrm{~V}$ and $\varphi=-15 \mathrm{~V}$. Once the analyser measurement deviation corrected, results are similar for the three potential biases (cf. FIG.11b). Indeed, it a maximum deviation is of $6.3 \%$ is observed at $E_{0}=50 \mathrm{eV}$ between $\varphi=-5 \mathrm{~V}$ and $\varphi=-15 \mathrm{~V}$ and of $1.6 \%$ between $\varphi=-10 \mathrm{~V}$ and $\varphi=-15 \mathrm{~V}$.

Surface potential should not change the energetic behaviour of the surface. Consequently, $R_{E}\left(E_{0}\right)$ values should not differ as a function of it. As measurement 


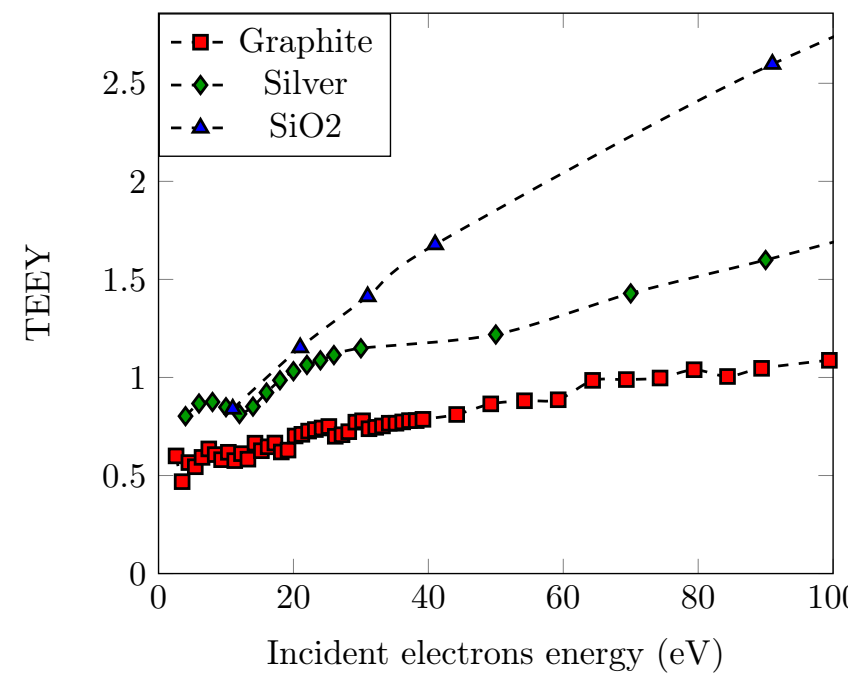

Figure 12: TEEY as a function of the incident electrons energy $\left(E_{0}\right)$ for three tested materials ( $\mathrm{SiO} 2$, graphite and silver) [28]

deviation has become negligible for values of $\varphi$ around $-10 \mathrm{~V}$, it is assumed that corrections and post-processes have allowed getting quantitative results. Nonetheless, it can be observed that measurement deviation is still important for $\varphi$ around $-5 \mathrm{~V}$. As $-5 \mathrm{~V}$ is a low surface potential, it may not be sufficient to prevent any emitted electron recollection by the sample, which could explain this deviation.

In the next section the results are shown for $\varphi=-9 \mathrm{~V}$ for practical reasons (accumulator potential difference). Nonetheless, as the potential impact on $R_{E}\left(E_{0}\right)$ measurement is negligible around $\varphi=-10 \mathrm{~V}$, the results stability is ensured.

\section{Results}

\subsection{TEEY and EEED measurements analysis}

FIG.12 shows TEEY for the three tested samples $\left(\mathrm{SiO}_{2}\right.$, graphite and silver). TEEY measurements of graphite and silver are reported from [28]. $\mathrm{SiO}_{2}$ measurements are reported from [29]. It can be observed that the three curves are increasing on the considered range of incident energy. However, one can notice that TEEY increases more sharply for $\mathrm{SiO}_{2}$ than for silver and graphite. Indeed, it was established that the TEEY of dielectrics material is generally higher than that of conducting ones due to the higher mean escape depth of the secondary electron in dielectrics materials [30. Besides, all along the energy range, the TEEY of graphite remains very low compared to the two other ones.

FIG 13 shows the measured EEED of a silver sample irradiated with an incident electrons beam of
$25 \mathrm{eV}, 50 \mathrm{eV}$ and $75 \mathrm{eV}$, before and after data correction explained in section 4.2. The emitted electrons energy distribution can be described throughout the example of FIG $13 \mathrm{~b}$ The peak situated at $50 \mathrm{eV}$ (after the correction that will be described in following section) is the elastically backscattered electrons (EBSE) peak. The quasi-Lambertian distributed curve observed at low energy (few eV), the secondary electrons (SE) distribution: the electrons issued and ejected from the material, the electrons at intermediate energy (between the SEs distribution and the EBSE peak) are the inelastically backscattered electrons (IBSE). According to FIG 13a, FIG 13b and FIG 13c, it can be noticed that the total transfer function has a non negligible impact on measured spectra proportions.

In FIG 14 , three spectra of emitted electrons are drawn for $E_{0}=25 \mathrm{eV}, E_{0}=50 \mathrm{eV}$ and $E_{0}=75 \mathrm{eV}$, after corrections. These spectra show that the elastic peak is moving away from secondary electrons peak when increases. Moreover, it can be noticed that, when $E_{0}$ increases, the elastically backscattered electrons ratio decreases, which is in agreement with experiments and models on BSE [31]. Finally, the ratio of emitted electrons mean energy on incident electrons energy decreases when $E_{0}$ increases (cf. FIG. 14 b in the top right corner).

\section{2. $R_{E}$ measurements analysis}

$R_{E}$ as the function of the incident electrons energy is shown in FIG 15a for $\mathrm{SiO}_{2}$, graphite and silver. $R_{E}$ varies significantly as a function of the incident energy and of the material. A sharp decrease at low $E_{0}$ is observed followed by a smoother decrease or a plateau region. For mono-energetic incident electron beam, a break in the slope is observed for the three materials. For silver, $R_{E}\left(E_{0}\right)$ experiences a sharp decrease from 0.80 to 0.40 for $E_{0}$ between $10 \mathrm{eV}$ and $50 \mathrm{eV}$, then a low growth from 0.40 to 0.48 between $50 \mathrm{eV}$ and $100 \mathrm{eV}$. For $\mathrm{SiO}_{2}$, a short decrease is observed from 0.68 to 0.57 for $E_{0}$ between $5 \mathrm{eV}$ and $22 \mathrm{eV}$, then a plateau is observed until $E_{0}=105 \mathrm{eV}$. For graphite, a stiff decrease is observed from 0.48 to 0.20 for $E_{0}$ between $5 \mathrm{eV}$ and 20 $\mathrm{eV}$, then a plateau is observed until $E_{0}=105 \mathrm{eV}$. One can notice that all the three materials follow a similar trend.

Graphite is the best absorber of incident energy with a plateau region at $R_{E}=0.2$, followed by silver with a lowest point at $R_{E}=0.4$ then $\mathrm{SiO}_{2}$ with a plateau region at $R_{E}=0.6$. Moreover, it can be observed that the sharp decrease is located between 0 and $20 \mathrm{eV}$ for $\mathrm{SiO}_{2}$ and graphite, 0 and $50 \mathrm{eV}$ for silver.

Nonetheless, in the context of HT, relevance of mono-energetic values can be questioned. It is more suitable to consider a given distribution of electrons arriving at the wall. In FIG $15 \mathrm{~b}$, results of 


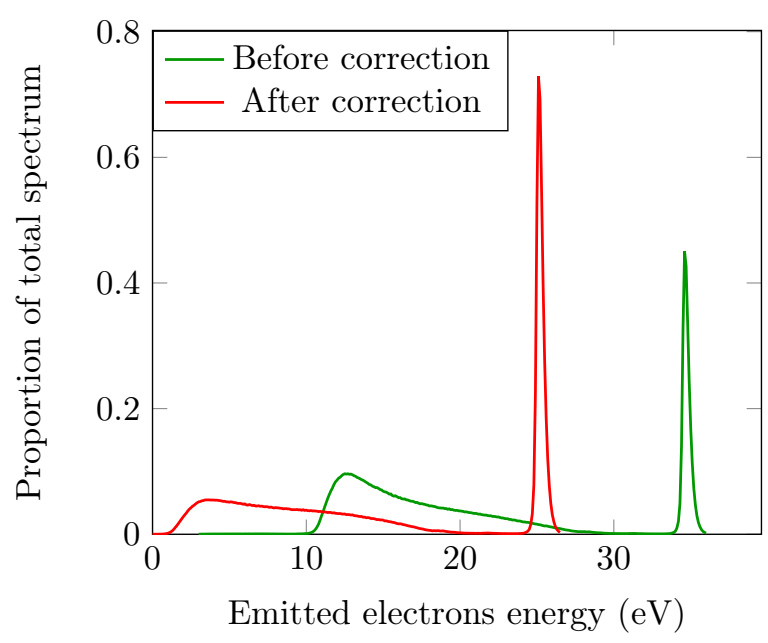

(a) Measured electron energy distribution of silver specimen for $E_{0}=25 \mathrm{eV}$ and $\varphi=-9 \mathrm{~V}$

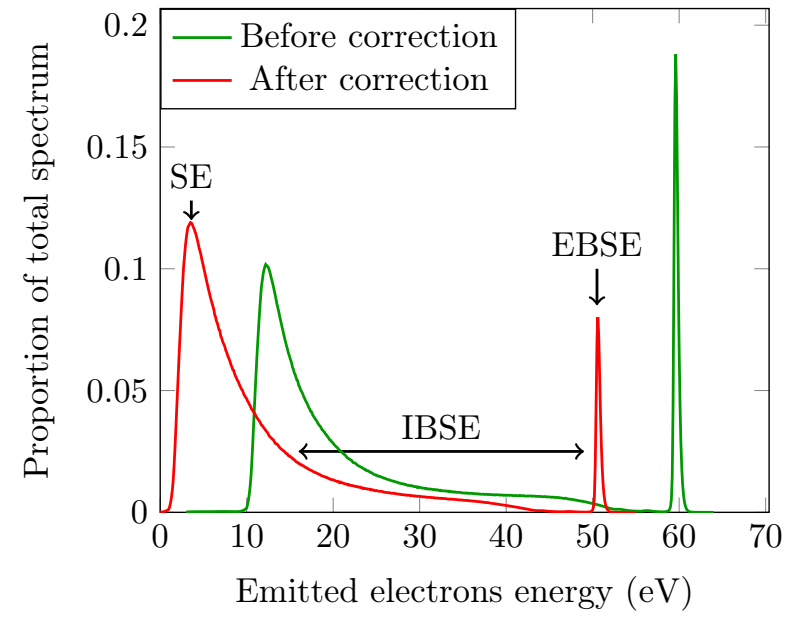

(b) Measured electron energy distribution of silver specimen for $E_{0}=50 \mathrm{eV}$ and $\varphi=-9 \mathrm{~V}$

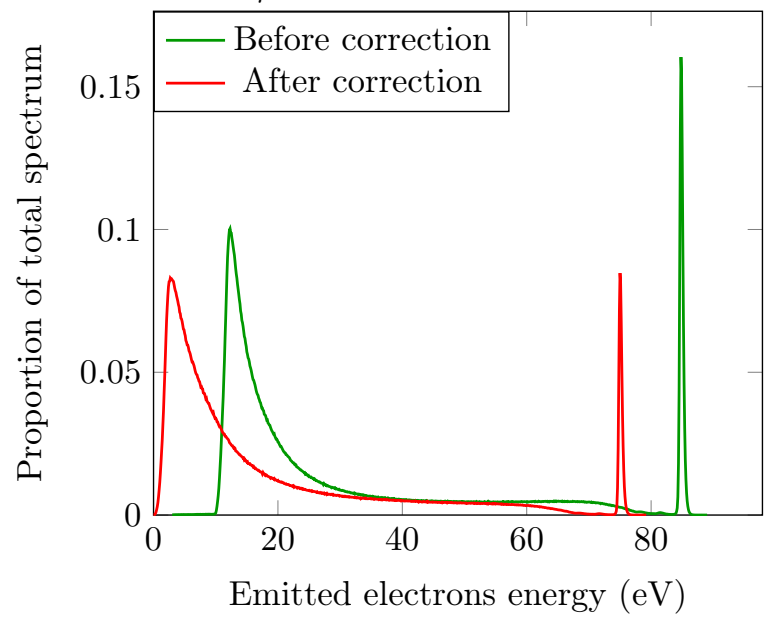

(c) Measured electron energy distribution of silver specimen for $E_{0}=75 \mathrm{eV}$ and $\varphi=-9 \mathrm{~V}$

Figure 13: Silver energy distributions from EA125 spectrometer for three incident electron energies $\left(E_{0}\right)$ and one surface potential $\varphi=9 \mathrm{eV}$. Results are presented before and after measurement biases correction

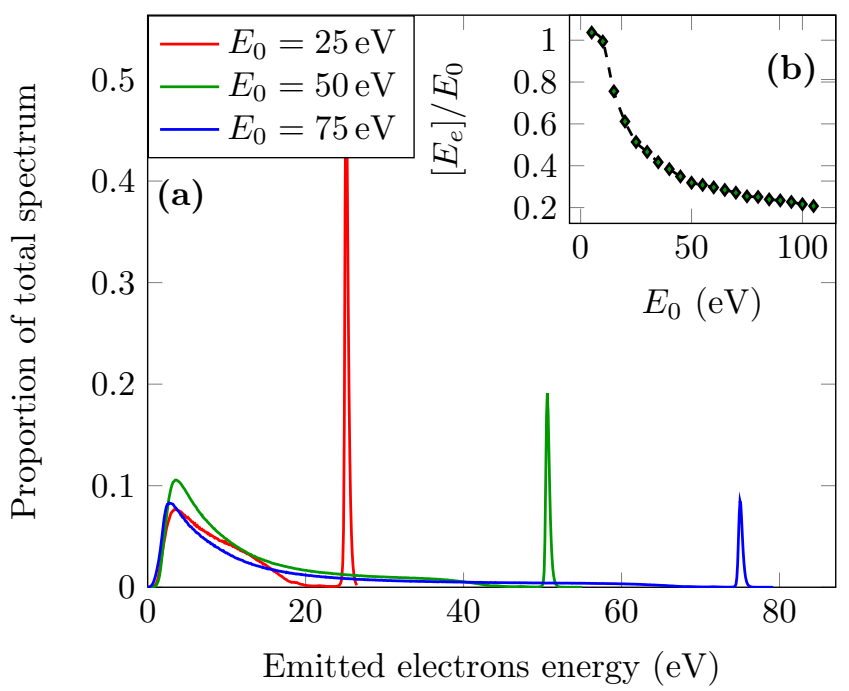

Figure 14: (a) Emitted electrons spectra for three incident electron energy $E_{0}$. (b) : Ratio of emitted electron energy on incident electron energy as a function of incident electrons energy

Table 1: Fitting parameters of $R_{E}\left(T_{e}\right)$ for a Lambertian distribution of electrons for three different materials $\left(\mathrm{SiO}_{2}\right.$, silver and graphite)

\begin{tabular}{lll}
\hline Material & $a[\varnothing]$ & $B\left[\mathrm{eV}^{-1}\right]$ \\
\hline Silver & -0.148 & 0.934 \\
$\mathrm{SiO}_{2}$ & -0.016 & 0.662 \\
Graphite & -0.283 & 0.758
\end{tabular}

FIG 15a have been integrated considering a Lambertian distribution of electrons (that is the distribution of a thermalised electrons cloud interacting with a surface) at a temperature $T_{e}$, as written in:

$$
\delta \Gamma_{\mathcal{L}, T_{e}}\left(E_{0}\right)=\frac{2}{\sqrt{\pi}} \sqrt{\frac{E_{0}}{T_{e}}} \exp \left(-\frac{E_{0}}{T_{e}}\right)
$$

The shape of $R\left(T_{e}\right)$ is the same for all the materials with a sharp decrease between 0 and $25 \mathrm{eV}$ and then a smoother decrease. In both case, it can be inferred that the change in slope is due a competition between the decrease of the emitted electrons energy on the incident electrons energy ratio (cf. FIG,14a) and the increase of TEEY (cf. FIG 12). These curves have been interpolated by a law of the following form:

$$
R_{E}\left(T_{e}\right)=B \cdot T_{e}^{a}
$$

This interpolation is valid for $T_{e}>5 \mathrm{eV}$. Fitting parameters $(a$ and $B$ ) are tabulated in Table 1 . 

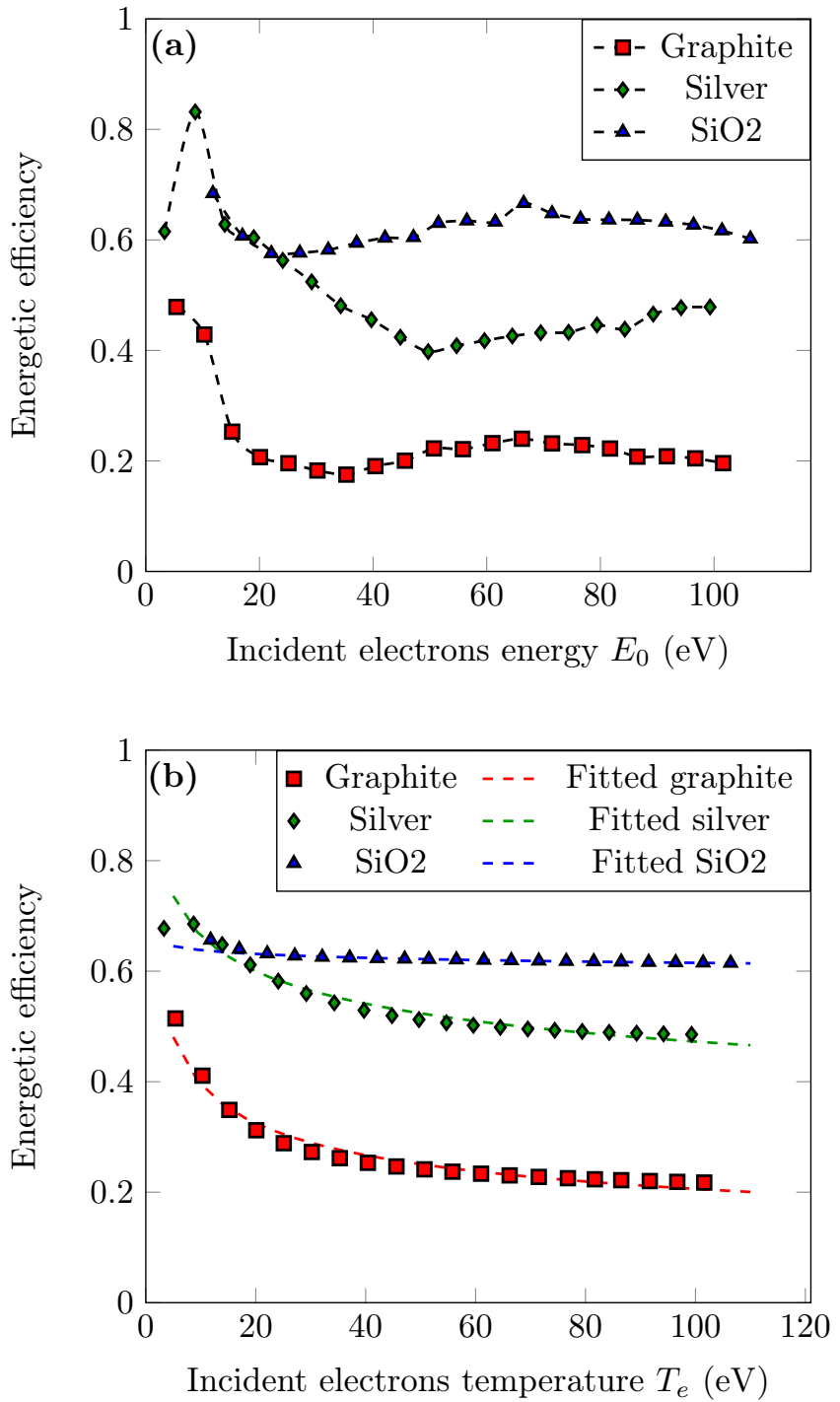

Figure 15: Measurements of $R_{E}$ as function of the incident electron energy for mono-energetic incident electron beam of energy $E_{0}[\mathrm{eV}](\mathrm{a})$ and for Lambertian distributed incident electron beam of temperature $T_{e}$ [eV] (b)

\section{Conclusions and further studies}

In this paper an experimental protocol to measure the energy balance of the EE at a wall submitted to an incident electrons flux has been presented. Then the need for EEED measurements corrections and post-process has been described. The corrections and post-processes are subsequently presented. Results on energy balance between incident electrons impacting a wall and reemitted electrons from that surface have been described. It has been shown that the energy balance is highly influenced by the energy of the impacting electrons and by the choice of the wall material. An analytical fit which can be used on fluid or hybrid models describing the discharge of HT has been proposed. Conversely, the knowledge of $R_{E}$ may allow a better prediction of multipactor phenomenon [32.

Further studies are in course to improve our knowledge on $R_{E}$ in more realistic operation conditions that involves the study of the effect of others parameters such as the wall temperature, angle between incident electron flux and wall surface, etc. Besides, $R_{E}$ analytical and numerical modelling are developed in parallel and have to be compared to these experimental data to check their validity. Development of analytical and numerical model of EE remains essential since certain parameters effects are not accessible to experiment. These experimental data need to be extrapolated by theory (for example, $R_{E}$ at razing angle).

\section{Acknowledgement}

Mr. Villemant benefits from an Onera/Cnes PhD. fellowship. Moreover, the authors are grateful to T. Gineste to allow using its calibration values and substantial experimental data for this work. This work has been carried out in the frame of the French jointresearch program Gis PPE. This work is supported by the CNES Research and Technology Program.

\section{References}

[1] E. M. Baroody. A Theory of Secondary Electron Emission from Metals. Phys. Rev., 78:780, 1950.

[2] A. J. Dekker and A. van der Ziel. Theory of the Production of Secondary Electrons in Solids. Phys. Rev., 86:755, 1952.

[3] R. G. Lye and A. J. Dekker. Theory of secondary emission. Physical Review, 107:977, 1957.

[4] G. F. Dionne. Origin of secondary-electron-emission yieldcurve parameters. Journal of Applied Physics, 46:3347, 1975.

[5] G. F. Dionne. Effects of secondary electron scattering on secondary emission yield curves. Journal of Applied Physics, 44:5361, 1973.

[6] C. Bouchard and J. D. Carette. The secondary electron emission coefficient of disordered surfaces. Surface Science, 100:241, 1980.

[7] M. Rösler and W. Brauer. Theory of Secondary Electron Emission. II. Application to Aluminum. Phys. stat. sol. (b), 104:575, 1981.

[8] M. Furman and M. Pivi. Probabilistic model for the simulation of secondary electron emission. Physical Review Special Topics - Accelerators and Beams, 5, 2002.

[9] N. Fil, M. Belhaj, J. Hillairet, and J. Puech. Multipactor threshold sensitivity to Total Electron Emission Yield in parallel-plate waveguide and TEEY models accuracy. In 2016 IEEE MTT-S International Microwave Symposium (IMS), page 1, 2016.

[10] K. C. Harkay. Theory and measurement of the electron cloud effect. In Proceedings of the 1999 Particle Accelerator Conference (Cat. No.99CH36366), volume 1, page 123, 1999. 
[11] N. Gascon, M. Dudeck, and S. Barral. Wall material effects in stationary plasma thrusters. I. Parametric studies of an SPT-100. Physics of Plasmas, 10:4123, 2003.

[12] D. M. Goebel and I. Katz. Fundamentals of Electric Propulsion. John Wiley\&Sons, INC., Hoboken, New Jersey, 2008.

[13] J. P. Boeuf and L. Garrigues. Low frequency oscillations in a stationary plasma thruster. Journal of Applied Physics, 84:3541, 1998.

[14] F. I. Parra, E. Ahedo, J. M. Fife, and M. Martínez-Sánchez. A two-dimensional hybrid model of the Hall thruster discharge. Journal of Applied Physics, 100:023304, 2006.

[15] D. R. Grosso, P. Barone, A. Bonanno, M. Camarca, A. Oliva, F. Xu, Lab Iis, and Dep Phys. "scrubbing" Process of $\mathrm{Cu}$ Surfaces Induced by Electron Bombardment. 2008.

[16] P. Riccardi, M. Pisarra, A. Cupolillo, M. Commisso, A. Sindona, R. A. Baragiola, and C. A. Dukes. Secondary electron emission spectra from clean and cesiated $\mathrm{Al}$ surfaces: the role of plasmon decay and data analysis for applications. J. Phys.: Condens. Matter, 22:305004, 2010.

[17] A. Héron and J. C. Adam. Anomalous conductivity in Hall thrusters: Effects of the non-linear coupling of the electron-cyclotron drift instability with secondary electron emission of the walls. Physics of Plasmas, 20:082313, 2013.

[18] Y. Raitses, I. D. Kaganovich, A. Khrabrov, D. Sydorenko, N. J. Fisch, and A. Smolyakov. Effect of Secondary Electron Emission on Electron Cross-Field Current in $E \times B$ Discharges. IEEE Transactions on Plasma Science, 39:995, 2011.

[19] L. Jolivet and J-F. Roussel. Numerical modeling of plasma sheath phenomena in the presence of secondary electron emission. IEEE Transactions on Plasma Science, 30:318, 2002 .

[20] M. D. Campanell, H. Wang, I. D. Kaganovich, and A. V. Khrabrov. Self-amplification of electrons emitted from surfaces in plasmas with $E \times B$ fields. Plasma Sources Sci. Technol., 24:034010, 2015.

[21] N. Hilleret, C. Scheuerlein, and M. Taborelli. The secondaryelectron yield of air-exposed metal surfaces. Appl Phys A, 76:1085, 2003.

[22] D. Payan N. Balcon. Secondary Electron Emission on Space Materials: Evaluation of the Total Secondary Electron Yield From Surface Potential Measurements. IEEE Transactions on Plasma Science, 40:282, 2012.

[23] J. Roupie, O. Jbara, T. Tondu, M. Belhaj, and J. Puech. The study of electron emission from aluminum in the very low primary energy range using Monte Carlo simulations. J. Phys. D: Appl. Phys., 46:125306, 2013.

[24] T. Gineste. Emission électronique sous impact d'électrons : applications spatiales. (in French). PhD thesis, 2015.

[25] M. S. Chung and T. E. Everhart. Simple calculation of energy distribution of low-energy secondary electrons emitted from metals under electron bombardment. Journal of Applied Physics, 45:707, 1974.

[26] M. Belhaj, O. Jbara, S. Odof, K. Msellak, E. I. Rau, and M. V. Andrianov. An anomalous contrast in scanning electron microscopy of insulators: The pseudo-mirror effect. Scanning, 22:352, 2000

[27] T Gineste. Personnal communication, 2016.

[28] M. Belhaj, J. Roupie, O. Jbara, J. Puech, N. Balcon, and D. Payan. Electron emission at very low electron impact energy: experimental and Monte-Carlo results. arXiv preprint arXiv:1308.1301, 2013.

[29] M. Belhaj, K. Makasheva, D. Teyssedreand, and D. Payan. Effect of charging on the secondary electron emission. Banff, Alberta, Canada, 2016.

[30] Jacques Cazaux. Some considerations on the secondary electron emission, $\delta$, from e- irradiated insulators. Journal of Applied Physics, 85:1137, 1999.

[31] A. Jablonski and P. Jiricek. Elastic Electron Backscattering from Surfaces at Low Energies. Surf. Interface Anal., 24:781, 1996.

[32] R. A. Kishek, Y. Y. Lau, L. K. Ang, A. Valfells, and R. M. Gilgenbach. Multipactor discharge on metals and dielectrics: Historical review and recent theories. Physics of Plasmas, 5:2120, 1998. 